nature in pediatric leukemia. Some of its elements were LSVs in DICER1 and NT5C2, known cancer genes. More broadly, the twenty most common B-ALL drivers (including NT5C2) showed higher prevalence of aberrant splicing than of somatic mutations. Thus, posttranscriptional deregulation of SF can drive widespread changes in B-ALL splicing and likely contributes to disease pathogenesis.

doi: http://dx.doi.org/10.7124/bc.0009C8

\section{Cancer-testis antigen MAGEA protein expression in extracellular vesicles released by cells}

\author{
Anneli Kuldkepp, Olavi Reinsalu, Magda \\ Karakai, Eve Toomsoo, and Reet Kurg \\ Institute of Technology, University of Tartu, Tartu, \\ Estonia \\ reet.kurg@ut.ee
}

To study the possible role of cancer testis antigens MAGEA proteins in cellular processes triggering normal cells to cancerous. The specific aim is to characterize MAGEA-positive extracellular vesicles (EVs). MAGEAs represent a unique class of tumor antigens which are expressed in a wide variety of malignant tumors, while their expression in healthy normal tissues is restricted to germ cells of testis, fetal ovary and placenta. Their restricted expression and immunogenicity make them ideal targets for immunotherapy in human cancers. MAGEA expression is observed mainly in cancers that have acquired malignant phe- notypes, invasiveness or metastasis, and the expression of MAGEA family proteins has been linked to a poor prognosis in cancer patients. MAGEA proteins have shown to interact ubiquitin ligases and regulate proteasome degradation of proteins, regulate the stability of p53 and interfere with the apoptotic pathway of the cell. Methods: MAGEA-positive EVs are isolated from cell culture media using differential ultracentrifugation and characterized by various biochemical and cellular methods, including western blot analysis, flow cytometry and confocal microscopy. Results: We show that MAGEA proteins are incorporated into extracellular vesicles of different sizes ranging from $150 \mathrm{~nm}$ to $2 \mu \mathrm{m}$ as well as to retrovirus Gag-induced virus-like particles (VLPs). MAGEA proteins, locating both in the nucleus and cytoplasm of the cell, are directed to the surface of EVs released by cells and furthermore, induce the shedding of cells. MAGEA proteins can also be used to guide recombinant proteins, e.g. EGFP and Cherry, onto the surface of EVs allowing to follow the behavior of EVs in real time. In addition, we show that MAGEA10 expression in mouse fibroblasts induces abnormal phenotypes, where the cell has multiple small nuclei. The mechanism of this phenomena and its biological significance is still not known and needs further investigations. Conclusions: We show that MAGEA proteins are incorporated into EVs and are exposed on the surface of EVs. This process is induced, at least to some extent, by the expression of MAGEA proteins itself and may have a role in formation of cancer metastasis. 